\title{
Low FODMAP diet \& prebiotic $\beta$-galactooligosaccharides improve irritable bowel syndrome and response to low FODMAP is predicted by urine and faecal metabolites: a randomised controlled trial
}

\author{
B. Wilson ${ }^{1}$, M. Rossi ${ }^{1}$, T. Kanno ${ }^{1}$, R. Hough ${ }^{2}$, C. Probert ${ }^{2}$, G. Parkes ${ }^{3}$, S. Anderson ${ }^{4}$, \\ P. Irving ${ }^{1,4}$, A.J. Mason ${ }^{1}$, M.C. Lomer ${ }^{1,4}$ and K. Whelan ${ }^{1}$ \\ ${ }^{1}$ King's College London, Department of Nutritional Sciences, London, UK, \\ ${ }^{2}$ University of Liverpool, Liverpool, $U K$, \\ ${ }^{3}$ Barts Health NHS Trust, London, UK and \\ ${ }^{4}$ Guy's and St Thomas' NHS Foundation Trust, London, UK.
}

This abstract was awarded the student prize.

Restricting oligosaccharides (low FODMAP diet, LFD) and conversely, supplementing with prebiotic $\beta$-galacto-oligosaccharides (B-GOS) have both been shown to reduce symptoms in irritable bowel syndrome (IBS) independently ${ }^{(1,2)}$. Notably, the LFD reduces faecal bifidobacteria, while B-GOS increases them. The combination of the two therapies has not previously been investigated. Further, identifying who will respond to a LFD would significantly advance patient care. The aims of this randomised controlled trial (RCT) were three-fold, investigating whether: (1) addition of $1.4 \mathrm{~g} / \mathrm{d}$ (active dose) prebiotic $\beta$-GOS (Bimuno, Clasado, UK) improves IBS symptoms alongside the LFD; (2) the dietary interventions alter fecal microbiota; and (3) urinary metabolites, faecal short-chain fatty acids (SCFA) or volatile organic compounds (VOC) can predict response to LFD.

Sixty-nine adults with IBS (Rome III) were recruited to a 3-arm RCT: control (sham diet/placebo), LFD (LFD/placebo) or LFD plus B-GOS (LFD + B-GOS) for 4-weeks. Global symptom question assessed 'adequate symptom relief' (response), and urine and stool samples were analysed at baseline and 4-weeks. Faecal microbiome were analysed using 16S rRNA MiSeq and QIIME software at baseline and 4-weeks, and Kruskal-Wallis used for taxonomic comparisons. Urine metabolomics ( $\left.700 \mathrm{MHz}{ }^{1} \mathrm{H}-\mathrm{NMR}\right)$, faecal SCFA (gas liquid chromatography) and faecal VOC (GC-mass spectrometry) were compared between responders and non-responders at baseline and 4-weeks. Urine metabolomic spectra and VOC profiles were analysed using principal component analysis and supervised pattern recognition methods orthogonal/partial least square discriminant analysis respectively. Faecal SCFA and VOC were compared using receiver operator characteristic (ROC) curves.

Response rate (adequate relief) at 4-weeks differed between control (30\%), LFD $(50 \%)$ and LFD + B-GOS $(67 \%)(p=.046)$, with post-hoc differences between control and LFD + B-GOS $(\mathrm{p}=0.015)$. The relative abundance of Actinobacteria differed significantly across groups at 4-weeks $(\mathrm{p}<0.001)$, specifically mean (SD) Bifidobacteria and Collinsinella spp were lower in the LFD (0.96 (1.03), $\mathrm{p}=0.015,0.50(0.56), \mathrm{p}=0.018)$, and LFD/B-GOS $(0.93((1.26) \mathrm{p}=0.002,0.44(0.34), \mathrm{p}=0.005)$ groups than control (1.97 (2.66), 1.13 (1.02) respectively). In the LFD group only, baseline measures were significantly different between responders and nonresponders in the urine metabolome $\left(Q^{2}=0.296\right.$ vs randomised -0.175$)$, and faecal VOCs $(A U C=0.854, p=0.045)$ and propionate (AUC $=0.848, \mathrm{p}=0.009)$ were predictors of response.

Combined LFD and B-GOS improves symptoms in IBS although B-GOS doesn't prevent the LFD reducing Actinobacteria. Metabolite profiling of urine and stool may predict response to the LFD and provide insight into mechanisms. Prospective clinical trials to test these algorithms are warranted and may lead to personalised therapy in IBS.

1. Schumann D, Klose P, Lauche R et al. (2018) Nutrition 45, 24-31.

2. Silk D, Davis A, Vulevic J et al. (2009) Aliment pharmacol ther 29, 508-518. 